\title{
LE PAYS COMME HÔTEL:TRANSCULTUREL ET MOBILITES GEO-SYMBOLIQUES DANS LES AMERIQUES
}

Patrick Imbert

\begin{abstract}
In the 19th century, writers in Québec and Mexico equated the United-States to a hôtel, that is to a place linked to change, instability and loss of identity. In Québec, in the 19th century, Adolphe-Basile Routhier, the author of the national anthem "O Canada" in its french version, valorized homogeneity and stability. These values are put in jeopardy by modernity and technology, a situation, which is seen as positive by the Canadian thinker T. C Keefer who published Philosophy of the Railroads in 1853. Nowadays, comparing a country like Canada to a hôtel is seen as very positive by thinkers such as Yann Martel and Pico Iyer. Both of them are keen suporters of transcultural encounters. Being a Canadian nowadays means valorizing change and hybridity.
\end{abstract}

Keywords: Transculturalism. Displacement.Hotel. Canadian Literature

\section{PAÍS COMO HOTEL: MOBILIDADES TRANSCULTURAIS E GEO-SIMBÓLICAS NAS AMÉRICAS}

Resumo: Análise dos fenômenos do deslocamento e transculturalismo através da imagem do "hotel" na literatura canadense. Como um cronotopo de passagem e instabilidade, o "hotel" presta-se para figurar relações que abrigam desejos de saberes múltiplos e ordena a liberação de temporalidades sem a rigidez dos fusos horários, permitindo a reescrita da história com inscrições lúdicas. No século XIX, os escritores mostravam o hotel como espaço de perda de identidade. Mas, no século XX, valores de homogeneidade e estabilidade foram questionados, uma situação vista como positiva pelo pensador canadense T. C Keefer que já havia publicado Philosophy of the Railroads em 1853, tratando do assunto. Atualmente, pensar o Canada como um "hotel" por escritores como Yann Martel e Pico Iyer significa acentuar as idéias de encontros transculturais, o que torna um canadense um ser imbuído de trocas e hibridismo.

Palavras-chave: Transculturalismo. Deslocamento. Hotel. Litaratura canadense. 
"Perhaps the most powerful cause of the breakdown of the closed society was the development of sea-communications and commerce". Karl Popper, The Open society and its Enemies, New York, Tavistock, 1963, p. 177.

\section{Introduction}

La Bible lue dans une perspective communautaire enracine dans un territoire restreint l'homme issu du sol et créé par Dieu. Il lui fait défendre un espace plutôt que de partir à la découverte du monde et de se considérer comme créé à partir d'un sol qui est la planète entière. Au Moyen-âge, la Chrétienté universelle, celle dont rêve en 1850 Monseigneur Bourget l'évêque de Montréal, se morcèle en États-nations sur la défensive face aux ennemis. C'est ce nouvel ordre issu d'Europe qu'il faut gérer et accomplir comme on le voit chez Jules-Paul Tardivel, auteur de Pour la patrie, publié en 1895 et proposant pour 1945, un Québec indépendant catholique, celui dont a rêvé Lionel Groulx. De nos jours, le rêve d'universalité fondé sur le religieux est repris par les fondamentalistes musulmans qui cherchent à inventer des réseaux transnationaux planétaires mais qui, temporairement, se contentent de tenter de réinstaurer au Levant un califat de style post-ottoman. Ils se réfèrent plus à un empire multiple centré sur le Moyen-Orient qu'à des Étatsnations voulus homogènes après avoir été constitués de façon arbitraire par les puissances coloniales.

Et la démocratie libérale dans tout cela? Depuis la fin de la deuxième guerre mondiale, ses 60 millions de morts, la Shoah, etc., elle tente de domestiquer la violence en favorisant le développement planétaire par la libéralisation du commerce, de l'échange des idées et de la circulation des personnes. Un grand mouvement de franchissement des frontières s'est progressivement mis en place qui a généré et génère angoisses, reculs et oppositions. La légitimité du déplacement, sa démocratisation sont pourtant en marche, car on sait que de tout temps, les gens riches, les nobles et les élites cultivées se sont déplacées géographiquement et symboliquement. Le mot taxi nous le rappelle. Ce sont les nobles de Thurn und Taxi qui ont créé un réseau de diligences qui sillonnaient l'Europe pour faciliter les échanges tandis que les serfs étaient attachés à la terre et vendus avec elle.

Ce mouvement de franchissement des frontières est perçu positivement par nombre de penseurs à toutes les époques, en particulier dans les Amériques, elles mêmes issues de la migration de la planète vers 
elles. Le Vénézuelien Fermin Toro $(1839,89)$ propose une constitution démocratique pour les États des Amériques. Elle devrait inclure le droit de se déplacer et de changer de pays. En 1853, le Canadien Anglais T. C. Keefer publie Philosophy of the Railroads. Il apporte des éléments sociologiques qui démontrent l'importance des déplacements et le fait que les trains vont changer le mode de vie des Canadiens en réduisant les distances qui se comptent maintenant en temps. C'est la durée qui devient importante et non l'espace qui sert à être franchi. Ainsi, les gens, dans les différentes régions du Canada, et partout progressivement dans les Amériques, vont s'habituer à vivre selon l'heure des fuseaux horaires et non selon l'heure locale. Voilà qui a pour corolaire, en 1885, le fait que le temps universel est établi à Greenwich en Angleterre, sous l'impulsion du Canadien Sandford Fleming. Le réseautage continental est en marche ainsi que celui de la planète. La pensée de Keefer reliant philosophie, sociologie, rationalisme et identité culturelle est adaptée à l'immensité du continent et à sa maîtrise comme à celle de la planète qui, progressivement, se rétrécit. Comme on le voit, les innovations technologiques jouent du mouvement et le favorisent. Elles se couplent à l'idée de légitimation et de facilité du déplacement, des rencontres, des communications interculturelles et du réseautage, ce qui propulsera le Canadien Marshall McLuhan à l'avant garde des penseurs mondialisés dans les années 1960.

Dans cette immense dynamique, nous allons nous intéresser à un des éléments de cette frénésie du déplacement et en considérer les marquages positifs et négatifs chez les penseurs des Amériques. Il s'agit de la résidence temporaire qu'est l'hôtel. Au $19^{\text {ème }}$ siècle et durant une partie du $20^{\text {ème }}$ siècle, dans les textes littéraires et à visées sociologiques, son image n'est généralement pas positive sauf aux États-Unis. Par contre, à la fin du $20^{\text {ème }}$ siècle et au $21^{\text {ème }}$ siècle, le pays est perçu lui-même comme un hôtel par certains penseurs comme Pico Iyer ou Yann Martel car ils sont ouverts au multiculturalisme, au transculturalisme et voient le changement d'un œil positif.

\section{1/ Le Canada français et les hôtels au $19^{\text {ème }}$ siècle.}

À la fin du $19^{\text {ème }}$ siècle, le montréalais Adolphe Basile Routhier critique la mobilité sociale, économique, spatiale et ethnique états-unienne : « 
À proprement parler, les États-Unis ne sont pas une patrie pour la plus grande partie de leurs habitants. C'est une immense hôtellerie où de nombreuses caravanes de peuples sont venues prendre un billet de logement » $(1871,86)$. À l'instar de l'Argentin Sarmiento affirmant que les citoyens des États-Unis ne disent jamais qu'ils sont pauvres mais qu'ils sont pauvres maintenant, ce qui relativise le processus d'attribution à visées essentialistes, Routhier est conscient du mouvement, du dynamisme et du changement de civilisation et d'épistémologie que représentent les États-Unis. Il est cependant en désaccord avec le mouvement, le melting pot et la venue d'immigrants qui contribuent rapidement au développement économique de cette hôtellerie que sont pour lui les États-Unis. Pour un tenant de la définition de la nation fondée sur le fait d'être un "enfant du sol » enraciné, ce mélange est inacceptable.

Certes des hôtels (Mueller 1998, 189-198) voient aussi le jour au Canada $^{1}$ car l'économie se développe au rythme de la technologie et des exportations mais, pour les penseurs canadiens français du $19^{\text {ème }}$ siècle, ils représentent une menace. En effet, ils sont les lieux de pénétration des publications américaines ${ }^{2}$ :

La littérature canadienne-anglaise [...] a la concurrence [...] de la presse à bon marché, des journaux illustrés, si nombreux chez nos voisins et de leurs reproductions des ouvrages anglais. Nos chemins de fer, nos hôtels, nos

\footnotetext{
1 "There is a superabondance of hotels in the neighbourhood of the Falls of Niagara", affirme James Stuart, Three Years in North America, Edinburgh, R. Cadell, 1833, p.158. Les visiteurs européens sont aussi fascinés par les hôtels à Montréal : " les plus beaux hôtels sont perdus au milieu des maisons secondaires » affirme Théodore Pavie, Souvenirs Atlantiques, Paris, Bossange, 1838, p. 157.

${ }^{2}$ Ce thème de l'influence des médias américains qui minent la culture canadienne sera une constante du paysage culturel canadien-anglais comme canadien-français et, de réglementations en déréglementations, diverses approches verront le jour suivant que l'on se référera à une politique de protection ou non. Ces médias états-uniens sont les véhicules privilégiés d'une culture où se mêlent le populaire et le spécialisé. Elle convient à des populations qui désirent se référer à certains mythes connus, mais transformés par les technologies modernes. Cette culture populaire/spécialisée est ce que les penseurs influencés par l'Europe appellent la culture de masse. Pourtant la culture de masse est beaucoup plus présente dans l'Union soviétique stalinienne et dans l'Europe nazifiée car il s'agit de régimes politiques centralisés, bureaucratiques, répressifs et qui ont voulu transformer les gens en masses soumises au chef et au parti. Cependant, par rapport à la conception européenne valorisant davantage l'écrit, la culture des États-Unis est plus fondée sur l'image et ses nouveautés technologiques, film, publicités ou image digitale, que sur la littérature. Au sujet de la littérature et de l'invention de l'identité nationale voir : Itamar Even-Zohar, "The Role of Literature in the Making of the Nations of Europe : A Socio-Semiotic Examination " in $A S / S A, \mathrm{n}^{\circ} 1$, 1996, p.1-11; http ://www.epas.utoronto.ca :8080/french/as-sa/ASSA-N*1/IEZ1.html
} 
Dans l'optique de Routhier, face aux États-Unis, le mot patrie n'est plus valide car le multiple des origines se conjugue à la frénésie de réussir économiquement. Ceci se produit souvent par le déplacement spatial comme par le déplacement des paradigmes pour des gens qui s'adaptent à la modernité commerciale propre à la logique démocratique états-unienne. Il ne faut pas oublier non plus l'attrait important des États-Unis sur les Canadiens français qui, de 1860 à 1900, émigrent très nombreux dans les villes manufacturières du Massachussetts ou du Rhodes-Island. C'est ce que rappelle Maurice Poteet (1987). Toute une campagne pour retenir les gens au Canada s'organise et elle se combine à la valorisation de la sédentarité comme on le verra aussi dans Maria Chapdelaine de Louis Hémon.

\section{2/ Les hôtels des États-Unis perçus par les voyageurs mexicains au $19^{\text {ème }}$ siècle.}

On trouve une attitude similaire chez les voyageurs mexicains aux États-Unis à la même époque. Ce qui choque le plus ces voyageurs qui partagent leurs impressions dans leurs récits de voyages aux États-Unis est le mouvement qui est lié au progrès et à la modernité. Déjà chez Manuel Balbotín, on s'étonne du fait que, dans les pensions, les gens ne se querellent pas avec la cuisinière, s'ils trouvent la nourriture peu à leur goût, mais qu'ils s'en vont ${ }^{3}$. La facilité du déplacement surprend surtout qu'elle est liée à une attitude où on n'entre pas en conflit. Ainsi, on se rend à une autre pension. Cet aspect souligne évidemment qu'il est possible de se déplacer facilement, car il n'y a pas de pénurie. Le mouvement est aussi souligné par Francisco Bulnes : « El Americano no puede vivir en un plano; tiene que imitar la Bolsa; ascender y descender; recibir en un piso, comer en otro, dormir más arriba,

\footnotetext{
${ }^{3}$ Voir Manuel Balbotín (1824-1894) dont un extrait du texte de 1873 intitulé Un día del mes de enero a los 40 grados de latitud norte est cité dans une collection de textes: Emmanuel Carballo, ¿Qué pais es éste? Los estados Unidos y los gringos vistos por escritores mexicanos de los siglos XIX y XX, México, Consejo Nacional para la Cultura y las Artes, 1996, p. 120. Merci à ma collègue Nora Guzmán de I'Instituto tecnológico de Monterrey pour m'avoir communiqué l'ouvrage de Emmanuel Carballo intitulé ¿Qué país es Éste?
} 
agitarse... ${ }^{4}$ " Bien sûr, ces jugements plutôt négatifs dans l'optique des voyageurs mexicains habitués, comme Routhier, à une société visant la stabilité, sont couplés à une rhétorique soulignant que la religion dominante aux États-Unis est l'énormité. Et les temples de l'énormité, en plus de la ville de New York tout entière, sont les hôtels. Ainsi, il semblerait que l'énormité et la facilité des déplacements soient les éléments constitutifs du progrès et qu'ils aient remplacé les valeurs religieuses fondamentales, l'humilité et l'attachement à un lieu communautaire. C'est ce que met en valeur Federico Gamboa utilisant l'image connue de l'Orient et des nomades orientaux pour dévaloriser les hôtels :

EL HOTEL WALDORF-ASTORIA EN NUEVA YORK. Caravansérail según el diccionario francés, quiere decir: 'parador público de Oriente, para alojar a las caravanas'. Enmiéndese la frase en el sentido de " parador público del oriente de los EE.UU. para alojar viajeros presuntuosos y marcadamente rastas...

Ces images font partie de l'arsenal rhétorique de tous ceux qui vivent le dualisme barbarie/civilisation issu de l'Europe et diffusé en Amérique latine par Sarmiento dans Facundo (1845). Sarmiento en applique le pôle civilisation à Buenos-Aires qui s'inspire d'un modèle européen pétrit d'aspiration à la stabilité ou de points de vue issus de pensées inspirées du monde aristocratique. Ces images manifestent que ces écrivains ne parviennent pas à prendre en charge les nouvelles dimensions territoriales comme économiques et culturelles du continent américain et de ses habitants qui ont participé à la révolution la plus importante du $19^{\text {ème }}$ siècle, celle de voter avec leurs pieds pour devenir des propriétaires et participer à l'expansion de leur monde comme de leur individualité. On prend

\footnotetext{
${ }^{4}$ Voir Francisco Bulnes (1847-1924) dont un extrait du texte de 1871 intitulé Sobre el hemisferio norte, 11, 000 leguas. Impresiones de viaje a Cuba, los Estados unidos, Japón, China, Egipto y Europa est cité dans une collection de textes: Emmanuel Carballo, ¿Qué pais es éste? Los estados Unidos y los gringos vistos por escritores mexicanos de los siglos XIX y XX, México, Consejo Nacional para la Cultura y las Artes, 1996, p. 151. Nous traduisons : L'Américain ne peut vivre sur un seul plan; il doit imiter la bourse; monter et descendre, recevoir sur un étage, manger sur un autre, dormir plus haut, s'agiter.

${ }^{5}$ Voir Federico Gamboa (1864-1939) dont un extrait du texte de son journal (1907-1938) intitulé Diario est cité dans une collection de textes: Emmanuel Carballo, ¿Qué pais es éste? Los estados Unidos y los gringos vistos por escritores mexicanos de los siglos XIX y XX, México, Consejo Nacional para la Cultura y las Artes, 1996, p. 179. Nous traduisons : L'HÔTEL WALDORF-ASTORIA À NEW YORK. Caravansérail selon le dictionnaire français, ce qui signifie : auberge publique de l'est des États-Unis pour loger les voyageurs présomptueux et notoirement rastas.
} 
connaissance de cette incapacité à comprendre tout le potentiel économique, technologique et culturel du continent et de ses habitants dans le rejet de l'arrière pays chez l'Argentin Sarmiento, le futur Président de la république (Andacht 2003). Dans cette optique, à l'instar de Fernando Gamboa, certains passages du texte Facundo présentent le paysage de la pampa comme un désert ${ }^{6}$ qui rappelle les déserts de l'Orient avec ses Bédouins ${ }^{7}$. C'est une image que reprend le monarchiste libéral Xavier Marmier dans ses Lettres sur I'Amérique de 1851 où il suit Sarmiento à la lettre dans ce domaine (Imbert 2010). L'on ne peut d'ailleurs s'empêcher de comparer leurs descriptions avec celles fournies par un Français, Théodore Pavie, en 1838, au sujet de Montréal:

[...] (du côté du nord-ouest de Montréal) ce ne sont que des forêts sauvages, monticules noirs et bleus comme le firmament, océan de bois, de déserts, de plaines comme les steppes de l'Asie, les sables de l'Arabie, puis tout cela s'enfonce et disparaît à l'ouest pour aller se perdre dans les glaces du pôle (p.163).

Mais Federico Gamboa ne se contente pas d'utiliser une rhétorique connue. Il affirme que l'hôtellerie se convertit en symbole des États-Unis tout entier, malades d'ostentation et pleins de nouveaux riches. Le déplacement représente le mal, car il est couplé à la mobilité sociale et aux transformations socio-culturelles. Gamboa rejoint ainsi Routhier qui tient à ce que les gens restent attachés à la terre et se contentent modestement de ce qu'ils ont comme le propose aussi dans sa postface à La terre paternelle Patrice Lacombe en 1845. C'est bien pourquoi les voyageurs Canadiens français comme Faucher de Saint-Maurice (Roger Le Moine 1995, 153) iront se battre pour l'empereur Maximilien au Mexique lors de l'invasion de ce pays par Napoléon III, car il leur semble au départ que les pauvres et les paysans mexicains combattant pour l'indépendance, sont des barbares d'un arrière pays à civiliser. Pour les Canadiens-français, dont certains ont combattus

\footnotetext{
${ }^{6}$ Cette rhétorique n'est pas complètement perdue comme le prouve un sous-titre d'un article récent concernant l'Argentine: «Entre la région de Buenos Aires, urbanisée et industrialisée, et le reste du pays, désert et sous-développé, il n’y a rien de commun.» Géo, avril 2002, p. 22. La multinationale Bertelsman qui publie ce magazine n'hésite pas à reconduire les vieux clichés.

${ }^{7}$ Ibn Khaldûn soulignait que les Bédouins étaient des nomades ennemis de la civilisation, des villes, de la pensée et de l'écrit. Voir à ce sujet: Olivier Rolin, Méroé, Paris, Seuil, 1998. On retient évidemment que pour tous ces penseurs, ce qui dérange, est la facilité du déplacement. Le nomadisme est senti comme une perte de contrôle, donc une brêche ouverte pour le chaos, grande peur des sédentaires et de ceux qui valorisent l'ordre et ses traditions.
} 
pour les Sudistes lors de la guerre de Sécession aux États-Unis, il faut établir une base aristocratique d'origine européenne au sud des États-Unis afin de contenir l'expansion territoriale, technologique et financière des États fédérés et contrôler la fascination qu'ils exercent sur les pauvres, les exclus et les ambitieux de la planète.

Il est donc clair que les voyageurs et les penseurs mexicains partagent un type de rhétorique avec Routhier. Celle-ci repose sur le rejet du mouvement, c'est-à-dire de la mobilité sociale et de la mobilité spatiale, deux éléments qui remettent en question la valorisation de l'enracinement national couplé au mythe biblique qui affirme que terre et signification sont indissolublement liés puisque l'homme est issu de la glaise originelle. Le nationalisme des penseurs canadiens et mexicains ne peut accepter la démocratisation de la mobilité même si celle-ci est à la base de la modernité et du progrès.

Plus tard, dans les années 1930, le rejet des hôtels des États-Unis, avec leur corolaire, mouvement, richesse, ambitions, se recontextualise en fonction de l'idéologie de gauche de certains artistes et intellectuels. En effet quand Diego Rivera et Frida Kahlo vont vivre à New York, car Diego est occupé à peindre, Frida à une vision négative des hôtels de la ville. Frida, comme le souligne J.M. G. Le Clézio dans son ouvrage intitulé Diego et Frida, ne parle pas anglais, à la nostalgie du soleil mexicain et de la population métissée et laborieuse. Elle s'ennuie et trouve que l'hôtel Barbizon-Plaza est un caravansérail froid et ennuyeux (113). Voilà qui se combine bien avec le fait que, depuis son voyage à San Francisco, elle ressent de l'hostilité envers les riches américains tandis que les autres, après le krach de 1929, sont dans le dénuement complet. Le symbole du mouvement, pour elle, ne se couple pas à l'ascension sociale comme le prouvent à cette époque les millions de chômeurs nourris dans les soupes populaires. Par contre, valoriser le nomadisme hôtelier se couple bien à une volonté d'imposer la reconnaissance de l'altérité dans une dynamique anticoloniale comme on le voit dans $L e$ Nègre de l'écrivain surréaliste français Philippe Soupault. Dans ce roman, il raconte l'histoire d'Edgar Manning, un Noir des États-Unis qui traverse l'Europe et les Amériques. Il vit soit dans des hôtels ou des logements temporaires, soit en prison. En note à ces aventures Soupault donne l'exemple d'un homme qui «Au lieu de parcourir les musées visitait les hôtels. Quand il ne pouvait plus trouver de nouvel hôtel, il quittait la ville » (1978 120). La 
légitimation du déplacement est totale et se couple d'un désir de changer la vie et de transformer la société.

\section{3/ Les transformations de l'hôtel dans la publicité au $20^{\text {ème }}$ siècle.}

De nos jours, par rapport à Routhier comme aux penseurs Mexicains, il se produit un renversement d'optique par rapport à l'hôtel. Le démontrent les nombreuses publicités des chaînes d'hôtels comme Hilton. Dans ces publicités, on montre d'immenses hôtels qui sont tous similaires et entourés d'un environnement limité qui ne permet pas de dire dans quel pays ils se trouvent. Ainsi, le mouvement impliqué par l'hôtel est-il malgré tout contrôlé par une structure architecturale, administrative et de services similaires en quelque lieu que ce soit de la planète. Le dépaysement dans le local n'a pas lieu, car une classe en processus de mondialisation va d'un point à un autre dans un univers prédéfini sous l'égide d'une marque qui fidèlise et protège, ce que souligne Pico Iyer dans The Global Soul quand il note que : "Lacking a binding sense of 'we', he (le citoyen contemporain) might nonetheless remain fiercely loyal to a single airline » $(2000,19)$. La mobilité se couple aussi à une nouvelle forme de stabilité par le partage d'un lieu favorisant les rencontres temporaires des membres d'une classe dirigeante élargie qui échappe temporairement, grâce au franchissement des fuseaux horaires, à la fois au local et au national pour s'engager dans le mondial. Ceci signifie que le lieu qui est environnement d'appropriation est en train de changer de signification. Il rejoint le concept d'espace qui est produit par le mouvement et les opérations. On se réfère ici à la distinction entre lieu et espace de Michel de Certeau dans L'Invention du quotidien (208). M. de Certeau suit la distinction saussurienne entre langue et parole et voit dans le lieu, la langue tandis que l'espace est l'expression de la parole. Dans notre contemporanéité en déplacement, cette distinction tend à se modifier car la langue, le lieu comme enracinement de l'appropriation est dépassé par l'espace comme créativité issue d'opérations pragmatiques.

\section{4/ L'hôtel et le " home ".}

Dans cette dynamique, il est intéressant de saisir que, récemment, certaines publicités cherchent à jouer de la reterritorialisation appropriative 
du lieu par le biais d'un humour qui joue de l'écart entre home et hôtel. Il faut bien voir d'ailleurs que cet écart se manifeste seulement entre la conception transportable du home anglo-saxonne ${ }^{8}$ et la conception de l'hôtel. Dans les cultures non anglo-saxonnes, le lien territoire/individu est très fort et ce jeu serait plus difficile comme un proverbe français l'affirme : on ne transporte pas sa terre natale à la semelle de ses souliers'. Par contre dans une culture anglo-saxonne, la contradiction n'est pas si forte car le home est un ensemble de structures culturelles et familiales transportables ${ }^{10}$. C'est ce que soulignait déjà G. Lévesque en 1848 :

Dans tous les cas, la patrie pour nous n'est pas ce qu'est le
home pour nos compatriotes d'origine anglaise et nous
aurons beau emporter avec nous notre trésor et
emmener notre famille et nous établir en pays étranger,
nous n'y trouverons jamais la patrie, tandis que leur
home peut les suivre partout puisque ce mot semble ne
pas comprendre le pays. (294); (dans James Huston, Le
répertoire national, $4^{\text {eme }}$ volume, (297).

Ces différences dans l'acception sont aussi soulignées dans le film mi-fiction, mi-documentaire "Home " de la réalisatrice québécoise Phyllis Katrapani diffusé en salle et sur internet, le mardi 27 août 2002, lors du $26^{\text {ème }}$ festival des films du monde de Montréal. Le titre de ce film en français est anglais, car, pour la cinéaste le mot home ne se traduit pas. Elle le traduit tout de même par chez soi. "Mais quand j'utilise le mot chez-soi, j'imagine quelqu'un qui est calé dans un sofa, avec un livre et un chat à coté de lui. Le concept du home, en anglais, est beaucoup plus vaste.» (La Presse, Mardi 27 août 2002, p. C3).

L'expansion hôtelière peut donc être perçue comme une version contemporaine de cette conception anglo-saxonne propre à la modernité qui

\footnotetext{
8 "Home is the only thing you do not leave home without " affirme Anton Shammas dans une conférence de 1994 (cité par Walter Mignolo, " Human understanding and (Latin) American Interests-The politics and Sensibilities of Geo-historical Locations ", in A Companion to Postcolonial Studies (ed. H Schwartz and S. Ray), Oxford, Blackwell, 1999, p. 182.)

${ }^{9}$ On peut le vérifier par la traduction de Homesick qui serait " avoir le mal du pays ", ce qui signifie non pas avoir des images de sa famille mais bien de la communauté et du territoire, du village niché au creux du vallon, etc.

${ }^{10}$ Cela ne signifie pas que l'enracinement n'est pas apprécié mais plutôt qu'il n'empêche pas le déplacement. Ainsi Mary Tolan, Global Managing Director de Accenture Resources affirme qu'elle a aimé, enfant, voyager avec son père qui savait apprécier la culture locale de la Forêt Noire. Toutefois elle souligne que sa famille a une propriété sur le lac Michigan depuis 1914 (Continental, August 2002, p. 33). La durée longue enracine.
} 
s'est développée dans l'extension de la frontier en tant qu'espace immense et ouvert et donc dont l'acception est l'opposée du terme français de frontière (Imbert 2017, 12-24). Cette expansion géo-symbolique se présente sous la forme d'un slogan accrocheur: «Is it possible TO BECOME HOMESICK for a hotel? " (Forbes, May 15, 2000, p. 55). Cette publicité pour Four Seasons Hotels and Resorts marque à la fois l'écart entre stabilité et mobilité et vise une fidélité de la clientèle qui, de manière symptomatique sur l'image, concerne une femme administratrice ou économiste. De ce fait, le mondial, déplace la stabilité centrée sur le home pour la reporter sur une sensation de bien être qui est liée au lieu de convergence de l'échange économique (une autre définition du home transportable) et qui n'est que rarement mis en place selon une relation propre à un international qui aurait un cachet local. En effet, le home est transportable et le cachet du home peut se diffuser mondialement.

Cependant certaines chaînes jouent aussi d'un enracinement qui n'est pas produit par le transport d'un environnement propre à la bourgeoisie internationale en circulation mais qui tient compte des esthétiques locales même si elles sont aseptisées. Ces chaînes qui ne sont pas d'origines nordaméricaines, jouent d'un rapport différentiel local/global plus marqué. "International Standards [...] Local Flavor » affirme la chaîne d'hôtels à capitaux hollandais Golden Tulip avec des photos de ses hôtels à Santiago, Miami, Trujillo (Perou) et Sao Paulo (American Way, May 15, 2000, p. 39).

Dans le cadre de cette compagnie s'affirme la différence qui ne fonctionne cependant que mieux si des standards internationaux sont en place $^{11}$. Grâce à ces deux exemples, on voit que le mouvement créant une allégeance renouvelée est le moteur du contemporain qui décontextualise/recontextualise les discours en jouant de deux codes complémentaires, mais selon des modalités différentes qui gardent en mémoire des différences culturelles d'origine. Dans le cas de la chaîne nordaméricaine, il s'agit d'un flux issu d'une culture où le home est mobile. Dans le cas de la compagnie européenne, il s'agit d'un double provenant d'une culture où la maison est liée en partie à un territoire. On semble promettre à une population en transit qui a en partie perdu son monde, un nouveau village

${ }^{11}$ Même chose pour la chaîne Taj de l'inde : « She has international business facilities but her hospitality is distinctly Indian » (Forbes, August 21, 2000, p. 113). 
globalisé. Toutefois, un hôtel concerne plutôt une communauté urbaine qu'un village, car il est réseauté au maximum. De plus, souvent, il est plus peuplé qu'un village. Dans ces exemples, se marquent clairement les modalités diverses des discours mondialisants qui ne tendent pas, même pour des multinationales de la prise en charge du déplacement, à une homogénéisation mais à des convergences où se notent toujours des différences reconfigurées selon les enjeux contemporains.

Il est remarquable de trouver une troisième publicité qui joue entre ces deux dimensions. Elle provient de Sheraton Argentine et concerne une bonne partie de l'Amérique latine. D'une Amérique latine vue d'Amérique latine mais branchée sur les États-Unis et sur le monde. On y voit la photo de deux enfants souriant accompagnée du texte suivant : "Sí abuela, nosotros también te extrañamos. Estar aquí es tan bueno como quedarse en tu casa"

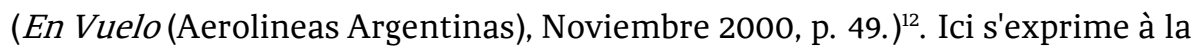
fois la référence à l'intime et l'international. Mais l'intime n'est pas défini comme le home anglo-saxon. Toutefois, le texte suggère que Sheraton est aussi convivial que la maison des grands-parents où accueil et permissivité sont de mise, plus que chez les parents. Il y a, sous jacent, un processus de transfert culturel qui tend à faire passer l'idée que l'hôtel pourrait aussi être un home et que cet espace convivial est transportable. Toutefois, il n'est pas possible d'affirmer ceci directement dans une société qui valorise fortement les relations familiales et l'insertion dans un lieu. Par conséquent, la publicité se contextualise avec cette structure culturelle argentine et le fait d'autant mieux que sa volonté de déplacer la signification du concept de maison est implicite. Elle reconnaît le point stable de la maison. Mais elle vise les nouvelles générations et resémantise les rapports petits-enfants/grandsparents. Elle déplace les significations en fonction d'un espace public international qui devient aussi attirant que le lieu convivial de la grand-mère. Déjà les enfants apprennent à être chez eux chez Sheraton, mais sans l'aïeule, ce qui est la première étape. La seconde sera la structure familiale immédiate. Autrement dit, cette publicité joue d'une part d'une sémantique qui reconnaît

\footnotetext{
12 Voir aussi la publicité de Marriott où on montre une famille avec deux enfants mis au premier plan mais sans insister comme dans la publicité Sheraton sur la destruction des liens entre enfants et grand parents (İcaro, Agosto 2002, p. 27). Nous traduisons ; Oui grand-mère, tu nous manques aussi. Être ici est aussi bien que rester chez toi.
} 
le stable et la valeur du local, tout en en déplaçant certains éléments. D'autre part elle aboutit à concevoir la maison comme un home, comme une structure se déplaçant, ce qui ouvre sur l'international déterritorialisé en contact avec le monde. "Quién cuida de usted? » nous demande-t-on à côté du logo « $S$ » de Sheraton. Bien sûr, c'est Sheraton qui s'occupe de nous ${ }^{13}$. Sans aller jusqu'à dire que parfois les résultats sont contradictoires comme le pense James Bohman $(1998,203)$, il reste néanmoins des liens familiaux et interpersonnels forts qui font que la maison ne peut être facilement déplacée dans le concept argentin et que donc la publicité Four Seasons concernant le sentiment d'être HOMESICK pour un hôtel serait inefficace dans le contexte latino américain.

Dans notre monde technologique réseauté, quand on veut présenter un lieu de passage comme un espace de permanence conviviale, on joue aussi à mêler le privé et le professionnel. Ceci permet de représenter l'hôtel comme un lieu où on se sent chez soi, car, comme à la maison, on utilise les mêmes outils technologiques et les mêmes programmes et on participe aux mêmes activités. C'est ce qui se passe dans les publicités Novotel visant le Mexique, en particulier la ville de Monterrey proche, à certains égards, de la culture étatsunienne qui voit l'hôtel comme l'expression du home transportable: "Novotel. Bienvenido- esta es su casa » (Escala (Aeromexico), Noviembre 2002, p. 93 $)^{14}$. On nous montre un père et son fils utilisant un ordinateur portatif pour jouer ensemble dans la chambre. Se met en place la dynamique du réseautage qui marque la capacité à l'accès.

La dynamique du réseautage tend à transformer le territoire en espace. C'est ce que marque très bien un romancier comme Pico Iyer dans The Global Soul où un producteur de film demande : “'What happened to home ?' The only home he knew, he said, had come in two unexpected moments of stillness-spiritual epiphanies, really- while travelling through rural Ireland"

\footnotetext{
${ }^{13}$ Nous traduisons: "Qui s'occupe de toi? ». On découvre une dynamique similaire dans un quotidien comme La Nación qui publie une section économique intitulée The Wall Street Journal Americas. On y découvre par exemple des informations mondiales et des informations plus locales concernant le Vénézuela, le Brésil, l'Uruguay en espagnol et sous format identique au Wall Street Journal des ÉtatsUnis : voir par exemple La Nación, Buenos Aires, viernes 10 de Noviembre de 2000, sección 2, p. 7, The Wall Street Journal Americas.

${ }^{14}$ Nous traduisons: « Novotel. Bienvenue-voilà ta maison. »
} 
$(15)^{15}$. Ce déplacement du concept de lieu se marque aussi dans le chapitre de The Global Soul consacré à l'aéroport de Los Angeles. Pour Pico Iyer, l'aéroport et l'urbain contemporain se rejoignent, car ils sont tous deux "a place where everyone's a stranger, so it seems, on his way to somewhere else" $(44)^{16}$, "the product ...of a mixed marriage between a border crossing and a shopping mall" $(49)^{17}$. Où se trouve alors la stabilité, sinon dans l'hôtel international branché sur les réseaux de fibres optiques permettant d'avoir accès à toutes les activités et à toutes les connaissances comme depuis le bureau ou la maison. La dynamique de réseautage manifestée dans la publicité du Novotel de Monterrey transforme l'aspiration à l'enracinement dans un lieu convivial en l'excitation de se connecter à des espaces différents en se vivant dans un module ouvert au concept de home comme bureau/maison transportable mais éloigné des complexités parfois chaotiques de la frontière ou du centre commercial.

\section{5/ Réseautage et légitimité du déplacement : le pays comme hôtel.}

Récemment, toutefois, c'est-à-dire à la fin du $20^{\text {ème }}$ siècle et au $21^{\text {ème }}$ siècle, voir le pays comme un hôtel devient tout à fait positif contrairement aux voyageurs mexicains ou à Adolphe-Basile Routhier. On comprend que les hôtels ont joué un rôle important dans la vision que les Canadiens ont eu d'eux-mêmes. N'oublions pas non plus les motels qui ont été tellement populaires au Canada, aux États-Unis et au Québec. Ainsi, Olga DuhamelNoyer note dans son ouvrage illustré de nombreuses photos intitulé Motel univers que "dans les grandes lignes, pour ce qui est du déployment motelier, le Québec va se comporter à la manière d'un État américain” (2006, 35). Jane Urquhart, quant à elle, a publié un livre de fragments intitulé $A$ Number of Things: Stories of Canada Told Through Fifty Objects où elle analyse la portée de différents objets historiques. Elle retient le Fairmont Empress Hotel à Victoria en Colombie-Britannique. Elle note qu'il s'y trouve une splendide

\footnotetext{
${ }^{15}$ Nous traduisons : Que s'est-il passé pour le chez soi ? Le seul chez soi qu'il ait connu, dit-il, s'est présenté durant deux moments inattendus d'épiphanies spirituelles et de calme, en fait - tandis qu'il voyageait dans l'Irlande rurale.

${ }^{16}$ Nous traduisons : un endroit où tout le monde est un étranger, à ce qu'il semble, en route pour aller ailleurs.

${ }^{17}$ Nous traduisons : le produit ...d'un mariage mixte entre un poste frontière et un centre commercial.
} 
peau d'un tigre du Bengale. Ce symbole de l'Empire et de l'importance de la colonisation de l'Inde rappelle un passé récent où le Canada dépendait fortement de l'Angleterre. Il est déplacé dans le roman de Yann Martel, L'histoire de Pi où un jeune Indien nommé Pi qui émigre au Canada avec sa famille se retrouve en plein océan avec un tigre du Bengale sur un radeau. L'histoire, au lieu de tourner à l'avantage du tigre, raconte leur coexistence multiculturelle avant de parvenir à atteindre les côtes mexicaines, puis Toronto pour le jeune Indien. L'Empire et son passé de domination anglaise sont recontextualisés dans une survie efficace mettant en valeur la rencontre dans la coopération favorisant le déplacement. Personne n'a la peau de l'autre. D'ailleurs, Pi aboutit à Toronto et il réussit à vivre une vie multiculturelle après cette expérience avec l'altérité radicale (Imbert 2014).

À partir de là, Yann Martel, en commentant son attitude d'ouverture à l'altérité a décrit le Canada comme "the greatest hotel on earth"18. Pico Iyer a simultanément proposé une comparaison similaire dans Imagining Canada: An Outsider's Hope for a global Future: "For her grateful immigrants fleeing Nazi Europe and first arriving in Canada, it seems like a luxury hotel, an oasis of ease and abundance; and a hotel, I think, is not such a terrible way of thinking about society: all its inhabitants in their own separate, confortable, well-serviced spaces, yet within common areas downstairs for dining, dancing and attending to civic responsibilities" (2001 $24)^{19}$. Iyer comme Martel présente cette comparaison comme un élément tout à fait remarquable et positif. En effet, grâce à ses politiques multiculturelles, celles définies notamment par Will Kymlicka dans Multicultural Odysseys: Navigating the New International Politics of Diversity, le Canada "welcomes people from everywhere" (Loo, January 29, 2017). Cela ne pose d'aillleurs pas de problème si les écrivains immigrants préfèrent résider au Canada tandis que leur imagination brode encore des histoires qui sont situées dans les pays qu'ils ont laissé derrière eux comme c'est le cas pour Michael Ondaatje, Ying

18 Tina "Hotel Canada", Canada's History. (http://www.canadashistory.ca/Magazine/Columnists/August-2011/Hotel-Canada, consulté le 29 janvier 2017).

${ }^{19}$ Nous traduisons: Pour ses (ceux du Canada) immigrants reconnaissants fuyant l'Europe nazie et arrivant d'abord au Canada, le pays à l'air d'un hôtel de luxe, une oasis de facilité et d'abondance, et un hôtel, je pense, n'est pas une manière si terrible de penser au sujet de la société. Tous ses habitants sont dans leurs propres espaces séparés, confortables et avec tous les services et ont accès à des espaces communs en bas pour manger, danser et participer aux responsabilités civiques. 
Chen, Dany Laferrière ou Daniel Castillo Durante. Pour ce dernier, Klaus Ertler (2013, 329) souligne que les personnages vivent un long désenchantement lié à l'exil et pas une renaissance comme pour Ying Chen ou Dany Laferrière. De plus, il faut retenir avec Klaus Ertler que de nombreux écrivains nés au Canada publient souvent des oeuvres qui se situent aussi aux antipodes. À l'instar de L'Orangeraie de Larry Tremblay qui est né à Chicoutimi, elles forment un corpus de textes liés à "la nouvelle écriture 'globalisante"' (Ertler 2017, 389).

Ainsi, on peut apprécier des auteurs qui s'inscrivent en partie dans des fictions travaillant la nostalgie ou un sentiment d'exil sinon de ressentiment comme Rawi Hage dans Cockroach. Toutefois, la plupart du temps, les auteurs arrivés récemment au Canada trouvent aussi souvent leur inspiration dans des dynamiques intégrées dans les rapports socio-culturels dans le contexte canadien comme c'est le cas pour Kim Thúy ou Dany Laferrière. Voilà qui amène à l'exploration d'images de soi multiples dans le multiple des caméléonages.

Comme le souligne Yann Martel dans Self ou dans Life of Pi, nous ne sommes pas membres d'une seule communauté. Nous pouvons couper nos attachements et en établir d'autres ou bien nous pouvons cumuler les attachements et les cultures. S'insérer dans une autre culture, apprendre une autre langue, ce n'est pas perdre ce que l'on sait déjà. La vie n'est pas un jeu à somme nulle (Imbert, 2013), surtout du point de vue culturel. Apprendre une langue, ce n'est pas perdre la langue première. Cela peut arriver dans des contextes minoritaires bien précis, c'est-à-dire dans le contexte d'un étatnation qui impose la langue dominante et méprise les autres. Mais dans le contexte de la mondialisation, toute langue, est utile pour renforcer les échanges et la compétition surtout quand on a, comme $30 \%$ de la population, eu accès à des études collégiales ou universitaires. Dans ce contexte, le rôle de l'échange culturel et de la légitimité des déplacements géographiques et symboliques est de permettre l'épanouissement d'un individu vivant un libéralisme pratique et circulant parmi les différences telles qu'elles s'expriment dans les grands centres urbains de la planète: le Mexico dont parle García Canclini (1995) par exemple où se croisent des modes de vie ruraux traditionnels, des modes de vie artistes et bohèmes, des modes de vie économico-technologiques Cette dynamique est elle aussi étudiée par Doug Saunders dans Arrival City (2010). Il montre la mobilité des gens 
nouvellement arrivés à Toronto. Ils ne restent que quelques années dans les quartiers immigrants pauvres et parviennent ensuite à se disperser dans de meilleurs quartiers dans la ville ou ailleurs au Canada. La culture a maintenant pour rôle de construire ce qu'un gouvernement ne peut faire: donner des buts communs dans le temporaire de rapports à construire en fonction d'imaginaires tournés vers l'avenir et le non-exclusif ${ }^{20}$. Le rôle du gouvernement et de sa bureaucratie devrait alors surtout être de s'assurer que des gens de cultures différentes et de plus en plus en contact proche par la migration, les échanges, le travail ou par internet, vivent en paix et aient un effet véritable, réciproque et positif les uns sur les autres. Ceci peut se faire en mettant en place des lois, des chartes et des règlements donnant l'occasion d'aboutir à une allégeance minimale fondée sur des textes et impliquant des droits et des marges de manœuvres redéfinissant la territorialité et le mythique, le tout couplé à une éducation scolaire et médiatique générant la coopération et la reconnaissance.

\section{Conclusion}

La transculture permet alors une reconnaissance entre gens capables de jouir de façons de vivre encore non-pensées en fonction de contextualisations qui ne sont inscrites ni dans la nature, ni dans l'histoire, mais dans un choix humain provenant de la capacité réflexive analysant les rapports de pouvoir comme les processus de codifications, les processus de production de discours et de croyances. Cette capacité est de plus en plus démocratisée sous forme d'incitation à devenir des producteurs de significations diversifiées trouvant place dans la littérature, les arts et les publicités (Imbert, 2003).

D'un certain point de vue, n'est ce pas ce que propose Laura Esquivel dans La ley del amor quand, dépassant le roman de la route explorant la diversité continentale États-Unis et Mexique, style Jack Kerouac, elle offre l'expérience d'une relecture du canon mexicain historique figé. Cette relecture passe par des réincarnations ludiques qui sont des accès pour

\footnotetext{
${ }^{20}$ Voir à ce sujet la distinction entre le substantive commitment impliquant de considérer les fins communes de la vie et le procedural commitment organisant les rapports sociaux sans considérations finalistes comme le souligne Charles Taylor dans Multiculturalism and the Politics of Recognition en 1993.
} 
revisiter l'histoire, pour vivre des rencontres redéfinies. Le roman se termine alors dans une parodie du consensuel communautaire. Ainsi, par cette parodie, Laura Esquivel indique que l'on peut s'ouvrir à une individualité libéralisée qui n'est ni essentialiste ni abstraite et universelle mais le fruit d'une conquête dans la visée vers un patrimoine culturel qui établit notre capacité de changer et de communiquer avec le différent. Dès lors, on peut penser que le territoire intérieur de chacun est aussi un hôtel qui abrite des désirs de savoirs multiples et qui comprend le franchissement de temporalités non plus marquées par les fuseaux horaires, mais par des réflexivités ludiques réécrivant l'histoire. Autrement dit, on se réfère à une mémoire qui se souvient des instants de bonheur comme des moments de violence et qui, au sujet de ces derniers affirme : Plus jamais ! Le transculturel est l'ouverture à la légitimité des déplacements dans la reconnaissance de la différence dans l'égalité. Il n'a rien à voir avec le relativisme.

\section{Bibliographie}

ANDACHT, Fernando (2003), "Global Symbols of the Latin-American Identity: winged Beings and Croissants with hot Chocolate" dans L'interculturel et l'économie à l'oeuvre (dir. D. Castillo-Durante et P. Imbert), vol. 3, Colección de las Américas/The Americas Series/Collection des Amériques, Ottawa, éditions David, p. 257-290.

BOHMAN, James (1998), “The globalization of the public sphere”, Philosophy and Social Criticism ", vol. 4, n*2/3 April 1998, p. 55-67.

CARBALLO, Emmanuel (1996), ¿Qué paìs es éste? Los estados Unidos y los gringos vistos por escritores mexicanos de los siglos XIX y XX. México, Consejo Nacional para la Cultura y las Artes.

CASTILLO-DURANTE, Daniel (2011), Le silence obscène des miroirs. Montréal, Lévesque.

CHAUVEAU, M. (1876), L'instruction publique au Canada. Québec, A. Core, 1876.

DE CERTEAU, Michel (1980), L'invention du quotidien. Paris, UGE/10/18.

DE SAINT-MAURICE, Faucher (1874), De Québec à Mexico. Montréal, Duvernay.

DUHAMEL-NOYER, Olga (2006), Motel univers, Montréal, Héliotrope.

ERTLER, Klaus-Dieter (2013), “Chassés-croisés canado-argentins. Le silence obscène des miroirs de Daniel Castillo Durante" dans Cultural Challenges 
of Migration in Canada/Les défis culturels de la migration au Canada. Frankfurt, Peter Lang, p. 317-331.

ERTLER, Klaus-Dieter (2017), “Trauma et identité de l'ailleurs: L'Orangeraie de Larry Tremblay". À la carte: Le roman québécois (2010-2015). Frankfurt, Peter Lang, p. 389-397).

ESQUIVEL, Laura (1996), The Law of Love. New York, Crown.

EVEN-ZOHAR, Itamar (1996), "The Role of Literature in the Making of the Nations of Europe : A Socio-Semiotic Examination » in $A S / S A, \mathrm{n}^{\circ} 1$, p.1-11; http ://www.epas. utoronto.ca :8080/french/as-sa/ASSA-N*1/IEZ1.html

GARCÍA-CANCLINI, Néstor (1995), Consumidores y ciudadanos. México, Grijalbo.

HAGE, Rawi (2010), Cockroach. New York, Norton and Company.

IMBERT, Patrick (2003), "Déplacements épistémologiques dans les littératures et les médias des Amériques ", L'interculturel au coeur des Amériques, (dir. D. Castillo-Durante et P. Imbert), Colección de las Américas/The Americas Series/Collection des Amériques, Université d'Ottawa / Université du Manitoba, Legas, p. 35-47.

IMBERT, Patrick (2010), De Canadá al Plata: Xavier Marmier y William Perkins" Los viajeros y el Río de la Plata: un siglo de escritura, coord. Jean Philippe Barnabé, Lindsey Cordery, Beatriz Vegh, Montevideo, Uruguay, Universidad de la República, p, 158-172.

IMBERT, Patrick (2013), Les Amériques transculturelles : les stéréotypes du jeu à somme nulle. Québec, Presses de l'Université Laval.

IMBERT, Patrick (2014), "Linking Transculturality and Transdisciplinarity", Semiotica, $n^{*} 202$, p. 571-587.

IMBERT, Patrick (2017), “Les territoires imaginaires de l'altérité : divers aspects de la frontier dans les Amériques", dans Interfaces Brésil/Canada, p. $12-24$.

IYER, Pico (2000), The Global Soul. New York, Vintage, 2000.

IYER, Pico (2001), Imagining Canada: An Outsider's Hope for a global Future. Toronto, The Hart House Lecture.

KEEFER, T. C. (1853), Philosophy of the Railroads. Montréal, Lovell, 1853.

KEROUAC, Jack (1972), Sur la route. Paris, Folio.

LAFERRIÈRE, Dany (1985), Comment faire l'amour avec un nègre sans se fatiguer. Montréal, VLB.

LE CLÉZIO, J.M.G. (1993), Diego et Frida. Paris, Stock. 
LE MOINE, Roger (1995), « L'aventure mexicaine de quelques Québécois (18641867) ", dans Les discours du nouveau monde au XIXe siècle au Canada français et en Amérique latine Los discursos del Nuevo Mundo en el siglo XIX en el Canadá francófono y en América latina, (ed. M. Couillard et P. Imbert) Ottawa, Legas, p. 253-262.

LÉVESQUE, G. (1848), "De l'influence du sol et du climat sur le caractère, les établissements et les destinées des Canadiens", Institut canadien de Montréal, 1848, p. 295-301, tiré de James Huston, Le Répertoire national, $4^{\mathrm{e}}$ volume.

LOO, Tina (consulté le 29 janvier 2017), “Hotel Canada", Canada's History. (http://www.canadashistory.ca/Magazine/Columnists/August-2011/Hotel-

Canada

MARMIER, Xavier (1851), Lettres sur l'Amérique. Paris, Bertrand, 1851 (traduites au Mexique: Cartas sobre la América, Mexico, imprenta del Universal, 185-, 2 vol.).

MARTEL, Yann (1998), Self. Montréal, XYZ.

MARTEL, Yann (2001), Life of Pi. Toronto, Vintage.

MIGNOLO, Walter (1999), "Human understanding and (Latin) American Interests-- The politics and Sensibilities of Geohistorical Locations ", in A Companion to Postcolonial Studies (ed. H Schwartz and S. Ray), Oxford, Blackwell, p. 177-189.

MUELLER, Thomas (1998), " Hotelgeschichte und Hotelgeschichten », dans Natur, Räume, Landschaften (dir. B. Krause et U. Scheck), München, Judicium Verlag, p. 189-198.

ONDAATJE, Michael (1992), The English Patient, New York, Vintage Books.

PAVIE, Théodore (1838), Souvenirs Atlantiques. Paris, Bossange.

POPPER, Karl (1963), The Open society and its Enemies. New York, Tavistock, 1963

POTEET, Maurice (1987), Textes de l'exode, recueil de textes sur l'émigration des québécois aux États-Unis : XIXème et XXème siècles. Montréal, Guérin.

ROLIN, Olivier (1998), Méroé. Paris, Seuil, 1998.

ROUTHIER, Adolphe-Basile (1871), Causeries du dimanche. Montréal, Beauchemin et Valois.

SARMIENTO, Domingo Faustino (1934), Facundo. Paris, Stock (1 ${ }^{\text {ère }}$ éd. 1845).

SAUNDERS, Doug (2010) Arrival City : the final Migration and our next World. Toronto, Knopf. 
STUART, James (1833), Three Years in North America. Edinburgh, R. Cadell.

TAYLOR, Charles (1993), Multiculturalism and the Politics of Recognition. Princeton (NJ), Princeton University Press.

THÚY, Kim (2009), Rú. Montréal, Libre expression.

TORO, Fermin (1839), « Europa y América » dans La doctrina conservadora.

Caracas, Ediciones commemorativas des sesquicentenario de la independencia.

URQUHART, Jane (2016), A Number of Things: Stories of Canada Told Through Fifty Objects. Toronto: Patrick Crean Editions.

YING Chen, Quatre mille marches. Montréal, Boréal, 2004

Recebido em 29 de abril de 2018.

Aceito em 25 de maio de 2018. 